\title{
Tensor character of pore pressure/stress coupling in reservoir depletion and injection
}

Müller, B., Altmann, J.B., Müller, T.M., Weißhardt, A., Shapiro, S., Schilling, F.R., Heidbach, O.

Geophysical Institute of the University of Karlsruhe, Karlsruhe, Germany

CSIRO Earth Science and Resource Engineering, Kensington, Australia

Department of Geophysics, Free University Berlin, Germany

Institute of Applied Geosciences, University of Karlsruhe, Karlsruhe, Germany

Helmholtz-Zentrum Potsdam, Deustches GeoForschungsZentrum-GFZ, Potsdam, Germany

\begin{abstract}
The state of stress within and around a reservoir is a key parameter for fluid flow, fracture stimulation, design of wellbore arrays and wellbore stability. Therefore pore pressure induced stress changes (pore pressure/stress coupling) have immediate implications for the reservoir management. We analyze the effects of pore pressure changes on the individual components of the principal stress tensor and not only the minimum horizontal stress component. The results show that pore pressure stress coupling has a tensor character and can cause significant changes in the stress field within the reservoir as a function of distance to the injection point and tectonic regime. The tensor character of pore pressure/stress coupling leads to changes in the differential stress of the system which is essential for fault reactivation. It is demonstrated that injection as well as depletion can lead to fault reactivation, and that the rock stability depends on the tectonic regime and on the location with respect to the injection (depletion) point. Furthermore, the tectonic regime can be locally modified within the reservoir. Thus an improved understanding of pore pressure/stress coupling effects contributes to fault seal prediction, optimized placement of in-fill wells, stimulation operations and wellbore stability assessment.
\end{abstract}




\section{Introduction}

Hydrocarbon and geothermal reservoirs experience changes in pore pressure during production. Secondary production methods may be needed to keep up reservoir pressure. However, injection and depletion occur normally not homogeneously throughout a reservoir. Hence, changes in pore pressure $P$ spread through the reservoir or compartment with time, thus depend on the distance to the injection (production) well. We focus on the effect of pore pressure/stress coupling (Engelder and Fischer, 1994; Hillis, 2000), the change of total stress associated with the change in $P$. In the hydrocarbon industry the smaller horizontal stress $\sigma_{h}$ is considered because $\sigma_{h}$ can be deduced with methods such as hydraulic fracturing and leak off tests (Haimson, 1975). Observations show that $\Delta \sigma_{h} / \Delta P$ is on average 65\% (Teufel et al., 1991), and thus not negligible. According to Engelder and Fischer (1994), the total vertical stress $\sigma_{v}$ is given by the overburden and is assumed to be unaffected by changes in $P$.

In this work we develop a simple theoretical basis for understanding the state of stress within reservoirs during the processes of injection (depletion) and derive analytical expressions for the coupling between $P$ and all components of the total stress tensor. Pore pressure and stresses are calculated taking into account different tectonic regimes. For three scenarios we analyze the effect of pore pressure/stress coupling on fault reactivation for cohesionless and cemented faults.

\section{Pore Pressure and State of Stress}

Terzaghi (1943) showed for soil and unconsolidated rocks, that the effective stress changes are determined by the amount of pore pressure change. This concept is successful in the interpretation of injection induced seismicity, e.g. for secondary production methods or enhanced geothermal systems (Deichmann and Evans, 2007) where an increase in $P$ reduces the effective normal stress on the fault and increases the likelihood for failure. The theory of poroelasticity refined the effective stress concept. For saturated porous rocks the effective stress is

$$
\sigma_{n, \text { eff }}(r, t)=\sigma(r, t)-\alpha \cdot P(r, t)
$$

with the effective stress coefficient or Biot-Willis parameter $\alpha$. Substituting the effective stress law in the constitutive equations and combining it with the pressure diffusion equation leads to a coupling of pore pressure and stress. This means that $P$ does not only affect the effective stress but also the total stress. Engelder and Fischer (1994) and others explain the pore pressure induced changes of the minimum horizontal stress $\sigma_{h}$ by poroelastic effects and obtain a simple relationship under the assumption of equal horizontal stresses $\left(\sigma_{h}=\sigma_{H}\right)$, constant vertical stress $\sigma_{v}$ and no horizontal strains:

$$
\frac{\Delta \sigma_{h}}{\Delta P}=\alpha \frac{1-2 v}{1-v} \text {. }
$$

For $\alpha=1$ and a Poisson's ratio of $v=0.25$, Eq. 2 yields $\Delta \sigma_{h} / \Delta P=2 / 3$. This ratio has been observed in many reservoirs by repeated $P$ - and $\sigma_{h}$-measurements (Engelder $\&$ Fischer, 1994; Teufel et al., 1991).

\section{Tensor Character of Pore Pressure/Stress Coupling}

Our approach for the pore pressure/stress coupling ratio considers the effect of $P$ on all principal stresses, not only on $\sigma_{h}$. We consider a homogeneous, saturated poroelastic medium, and use the equations for the spatio-temporal pore pressure and stress distributions caused by continuous fluid injection (depletion) at one point (Rudnicki, 1986). We derive the long-term limits $(t \rightarrow \infty)$ of the pore pressure/stress coupling ratio $\Delta \sigma / \Delta P$ for the radial stress $\Delta \sigma_{\text {rad }}$ and the tangential stress $\Delta \sigma_{\text {tan }}$

$$
\lim _{t \rightarrow \infty} \frac{\Delta \sigma_{\text {rad }}}{\Delta P}=\alpha \frac{1-2 v}{1-v}, \quad \lim _{t \rightarrow \infty} \frac{\Delta \sigma_{\text {tan }}}{\Delta P}=\frac{1}{2} \alpha \frac{1-2 v}{1-v} .
$$

This means that in the long-term limit, the radial stress is affected twice as much as the tangential stress by a change in $P$. The principle stress tensor, after a pore pressure change $\Delta P$, is then given by: 


$$
\sigma_{i j}=\left[\begin{array}{ccc}
\sigma_{\text {rad }}+\alpha \frac{1-2 v}{1-v} \Delta P & 0 & 0 \\
0 & \sigma_{\tan }+\frac{1}{2} \alpha \frac{1-2 v}{1-v} \Delta P & 0 \\
0 & 0 & \sigma_{\tan }+\frac{1}{2} \alpha \frac{1-2 v}{1-v} \Delta P
\end{array}\right] .
$$

The effective principal stress tensor, after a pore pressure change $\Delta P$ has occurred, results into:

$$
\sigma_{i j, \text { eff }}=\left[\begin{array}{ccc}
\sigma_{\text {rad }}-\frac{1}{3} \Delta P & 0 & 0 \\
0 & \sigma_{\tan }-\frac{2}{3} \Delta P & 0 \\
0 & 0 & \sigma_{\tan }-\frac{2}{3} \Delta P
\end{array}\right] .
$$

Without consideration of pore pressure/stress coupling a $30 \mathrm{MPa}$ pressure drop in the reservoir would increase the effective principal stresses by $30 \mathrm{MPa}$, leading to fault stabilization. Depletion can lead to a significant reduction in $P$ in the order of 10's of $\mathrm{MPa}$, depending on production rate and time since start of production, e.g. $20 \mathrm{MPa}$ pressure drop from 1975 to 1990 in the Ekofisk field (Teufel, 1991). However, under consideration of pore pressure/stress coupling, a pore pressure reduction of $30 \mathrm{MPa}$ causes $\sigma_{\text {rad,eff }}$ to increase by $10 \mathrm{MPa}$ and $\sigma_{\text {tan,eff }}$ by $20 \mathrm{MPa}$. This results in a change in differential stress of maximal $10 \mathrm{MPa}$, depending on the tectonic regime and the position within the reservoir. Such differential stress changes are significant at reservoir depth, e.g. $\sigma_{\mathrm{v}} \approx 45 \mathrm{MPa}$ at $2 \mathrm{~km}$ depth.

\section{Implications for rock failure}

For failure the amount of differential stress is essential. To infer the effects of pore pressure/stress coupling and the associated differential stresses changes for reservoir injection (depletion), we analyze the stress state of the long-term injection (depletion) for different tectonic regimes. In the following we consider a hypothetical reservoir (Figure 1) in which we deduce the stress changes caused by pore pressure variations for long injection (depletion) times at 3 positions along the principal stress axes at equal distance to the injection (depletion) point P. Point $\mathrm{A}$ is located on the $\sigma_{H^{-}}$ axis, Point $\mathrm{B}$ on the $\sigma_{h}$-axis and Point $\mathrm{C}$ on the $\sigma_{\mathrm{v}}$-axis.

Figure 2 displays the effective stress states at positions $\mathrm{A}, \mathrm{B}$ and $\mathrm{C}$ by Mohr circles of different colours. The black solid line Mohr circles indicate the initial effective stress state, the black dashed line Mohr circles the effective stress states caused by injection (depletion) without considering pore pressure/stress coupling. The stress states at position A, B, C after injection (depletion) and taking pore pressure/ stress coupling into account, are given as blue, red and green Mohr circles. The longterm limit pore pressure change was assumed to be moderate $( \pm 15 \mathrm{MPa})$ and equal in all examples. Under consideration of pore pressure/stress coupling the differential stress, and thus the size of the Mohr circles can be constant, decrease or increase, dependent on tectonic regime and location with respect to the injection (depletion) point, but differs always from the uncoupled case (dashed line).

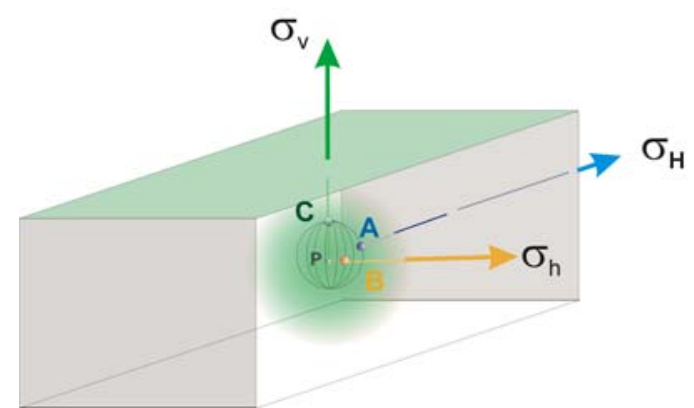

Figure 1 Points A, B, C located on principal stress axes $\sigma_{H}, \sigma_{h}, \sigma_{v}$. at equal distance to the injection point $P$. The shaded area sketches the pore pressure distribution which is radial-symmetric around $\mathrm{P}$.

Normal Faulting Regime (NF-regime, $\sigma_{\mathrm{v}}>\sigma_{\mathrm{H}}>\sigma_{\mathrm{h}}$ ): In the case of depletion, $\Delta P$ increases the effective minimum stress along the $\sigma_{h}$-axis by the amount of $|1 / 3 \Delta P|$ because $\sigma_{h}$ is the radial stress, whereas the effective vertical stress is tangential stress, and thus increased by $|2 / 3 \Delta P|$. Therefore, the 
reduction in $P$ increases the differential stress at position B (red Mohr circle). Similarly, a reduction in $P$ reduces the differential stress at point $\mathrm{C}$ (green Mohr circle). No change occurs along the $\sigma_{\mathrm{H}}$-axis, and thus at position A (blue Mohr circle). Reactivation of faults in NF-regimes due to depletion is most likely along the $\sigma_{h}$-axis. In the case of injection, the effective vertical stress reduces by $1 / 3 \Delta P$ at point $\mathrm{C}$, whereas both effective horizontal stresses reduce by $2 / 3 \Delta P$, what leads to larger a differential stress. At point $\mathrm{A}$, both the effective vertical and effective minimum horizontal stress are reduced by $2 / 3 \Delta P$, hence the size of the blue Mohr circle does not change. At point $\mathrm{B}$, the effective vertical and the effective maximum horizontal stress are reduced by $2 / 3 \Delta P$, the effective minimum horizontal stress by $1 / 3 \Delta P$, leading to lower differential stress. Thus, fault reactivation in a NFregime due to injection, is most likely along the $\sigma_{v}$-axis.

Strike Slip Regime (SS-regime, $\sigma_{\mathrm{H}}>\sigma_{\mathrm{v}}>\sigma_{\mathrm{h}}$ ): Injection into a SS-regime increases the differential

\section{Normal Faulting Regime - Injection $15 \mathrm{MPa} \quad$ Normal Faulting Regime - Depletion $15 \mathrm{MPa}$}

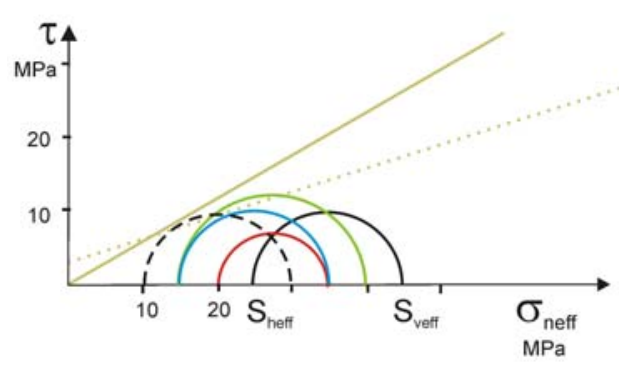

Strike Slip Regime - Injection $15 \mathrm{MPa}$

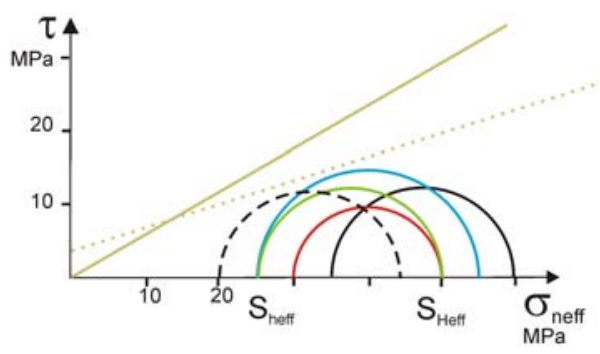

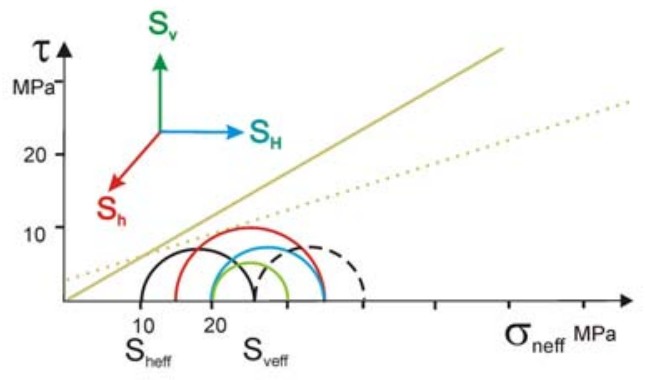

Strike Slip Regime - Depletion $15 \mathrm{MPa}$

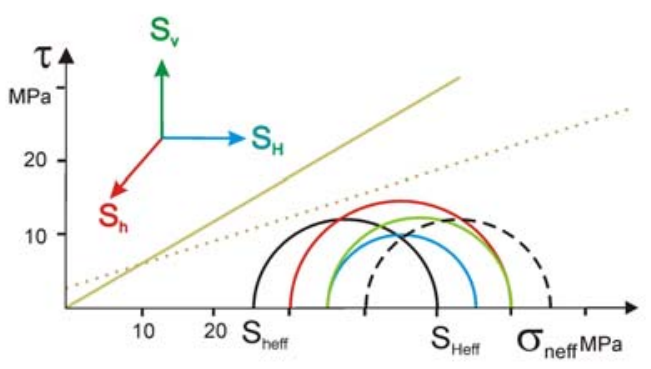

Thrust Faulting Regime - Injection $15 \mathrm{MPa}$

Thrust Faulting Regime - Depletion $15 \mathrm{MPa}$
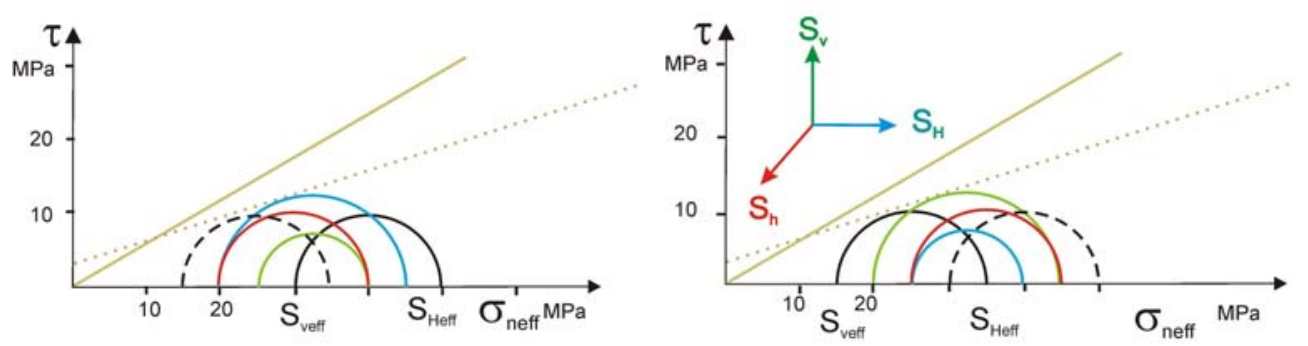

Figure 2: Effective stress states at positions A (blue), B (red), C (green) for injection (left column) and depletion (right column) in normal faulting $\left(\sigma_{v}>\sigma_{H}>\sigma_{h}\right)$, strike slip $\left(\sigma_{H}>\sigma_{v}>\sigma_{h}\right)$ and thrust faulting $\left(\sigma_{H}>\sigma_{h}>\sigma_{v}\right)$ regimes. Without pore pressure/stress coupling (dashed lines), the Mohr circles remain at the same size and are simply shifted along $x$-axis. Considering pore pressure/stress coupling the differential stress changes (blue, red and green Mohr circles). The two example failure envelopes represent cohesionless (solid lines) faults with a coefficient of static friction $\mu=0.6$, and cemented (dashed lines) faults with $\mu=0.35$ and a cohesion of $3 \mathrm{MPa}$. 
stress along the $\sigma_{H^{-}}$axis (blue Mohr circle), which brings faults located in $\sigma_{H^{-}}$-direction closer to failure, whereas faults along $\sigma_{h}$-axis can be stabilized (red Mohr circle). Depletion reduces the differential stress along the $\sigma_{H}$-axis (blue Mohr circle) and increases it along the $\sigma_{h}$-axis (red Mohr circle). In this case failure is most likely in direction of $\sigma_{h}$.

Thrust Faulting Regime (TF-regime, $\sigma_{\mathrm{H}}>\sigma_{\mathrm{h}}>\sigma_{\mathrm{v}}$ ): Failure is most likely above or below the depletion point. In contrary, injection causes the differential stress to increase along the $\sigma_{H}$-axis and to decrease along the $\sigma_{v}$-axis, which enables failure in direction of $\sigma_{H}$. The likelihood for reactivation depends on the state of stress, but also on the constraints of the fault. Cohesionless, critically stressed faults are most likely to be reactivated during injection because the mean effective stress is reduced. They are less sensitive in case of production, because the increase of mean effective stress counteracts the differential stress change. Concerning cemented faults with cohesion, pore pressure/stress coupling can reactivate faults also during depletion and cause leakage.

If two of the principal stresses are similar in magnitude prior to injection (depletion), the tectonic regime might be locally changed by injection (depletion) due to pore pressure/stress coupling. A change of the tectonic regime has significant effects on a number of geomechanical issues like wellbore stability, because the failure conditions around wellbores depend on the relative stress magnitudes (Fuchs and Müller, 2001). Also the orientation of hydraulically induced fractures is affected by local regime changes. In SS-regimes the fractures are vertical perpendicular to $\sigma_{\min }$, in TFregimes they might be horizontal because $\sigma_{v}$ is the minimum principal stress.

\section{Conclusions}

The filling of reservoirs depends on the seal capacity of the reservoir bounding faults and the cap rock. Our investigations contribute to understand potential seal or cap rock leakage. Our results show: Pore pressure affects all principal stress components. The stress components can be considered in terms of radial and tangential stresses with respect to the pore pressure cloud. The stresses induced by pore pressure changes modify the likelihood of fault reactivation. For injection in SS- and TFregimes, the likelihood for fault reactivation is greatest along the direction of $\sigma_{\mathrm{H}}$, in NF-regimes reactivation is most likely above or below the injection point. For depletion in NF- and SS-regimes, fault reactivation occurs most likely in $\sigma_{h}$-direction. In TF-regimes, reactivation is most likely in vertical direction. Cemented faults are more likely to be reactivated by pore pressure/stress coupling than cohesionless faults. Pore pressure/stress coupling can locally modify the tectonic regime, thus cause significantly different stress conditions as information of geomechanical models.

\section{References}

Deichmann, N. and Evans, K. [2007] Seismicity induced by water injection for geothermal reservoir stimulation $5 \mathrm{~km}$ below the city of Basel, Switzerland, AGU Fall Meeting.

Engelder, T. and Fischer, M.P. [1994] Influence of poroelastic behaviour on the magnitude of minimum horizontal stress, Sh, in overpressured parts of sedimentary basins. Geology, 22, 949-952.

Fuchs, K. and Müller, B. [2001] World stress map of the earth: a key to tectonic processes and technological applications. Naturwissenschaften, 88(9), 357-371.

Haimson, B. [1975] Deep in-situ stress measurements by hydro fracturing. Tectonophysics, 79, 41-47.

Hillis, R.R. [2000] Pore pressure/stress coupling and its implications for seismicity. Exploration Geophysics, 31, 448-454.

Rudnicki, J.W. [1986] Fluid mass sources and point forces in linear elastic diffusive solids. Mechanics of Materials, 5, 383393.

Terzaghi, K. [1943] Theoretical soil mechanics. John Wiley, New York.

Teufel, L., Rhett, D. and Farrell, H. [1991] Effect of reservoir depletion and pore pressure drawdown on in situ stress and deformation in the Ekofisk field, North sea. In Rogiers, J. (Ed.) Rock Mechanics as a Multidisciplinary Science. Balkema, Rotterdam, 63 Ü72. 